\title{
Using National Early Warning Score (NEWS) 2 to help manage medical emergencies in the dental practice
}

Phil Jevon*1 and Shaam Shamsi

\section{Key points}

Provides information about National Early

Warning Score (NEWS) 2
Discusses benefits of using NEWS2 in the dental practice.
Discusses how to introduce NEWS2 into the dental practice.

\begin{abstract}
If a medical emergency occurs in the dental practice, members of the dental team must be able to respond promptly, effectively and safely. Fundamental to this response is knowing when it is necessary to call 999 for an ambulance and communicating effectively with the ambulance service to ensure the timely arrival of the emergency services and timely transfer to hospital. This can be helped by using the Royal College of Physicians' (RCP's) National Early Warning Score (NEWS) 2, widely used by the ambulance service and in hospitals - it reliably detects deterioration in adults, triggering review, treatment and escalation of care. Although NEWS2 hasn't yet been validated for use in primary care, NHS England is encouraging its widespread use in this sector. Using NEWS2 in the dental practice will help the dental team to effectively, confidently and safely manage medical emergencies, including sepsis, should they arise. This will facilitate effective teamwork and help to ensure enhanced patient outcomes. This article provides an overview of NEWS2, including benefits for using it in the dental practice and guidance on how to implement it.
\end{abstract}

\section{Introduction}

Members of the dental team are expected to be able to effectively and safely manage medical emergencies in the dental practice. ${ }^{1}$ Fundamental to achieving this is recognising when patients are ill and communicating effectively with the ambulance service to ensure the timely arrival of the emergency services and timely transfer to hospital.

The Royal College of Physicians' (RCP's) National Early Warning Score (NEWS) 2, widely used in the healthcare setting both in the UK and abroad, reliably detects deterioration in adults, triggering review, treatment and escalation of care. ${ }^{3}$ Although NEWS2 has yet to be validated for use in primary care (research is ongoing), NHS England is encouraging its use in this sector. ${ }^{3}$

${ }^{1}$ Academy Tutor, Walsall Teaching Academy, Manor Hospital, Walsall, UK; ${ }^{2}$ Director, Horizons Dental Centre, Staffordshire, UK

*Correspondence to: Phil Jevon

Email address: phil.jevon@walsallhealthcare.nhs.uk

Refereed Paper.

Accepted 22 May 2020

https://doi.org/10.1038/s41415-020-2024-6
The aim of this article is to understand how NEWS2 can help members of the dental team manage a medical emergency in the dental practice.

\section{Background}

NEWS was developed in 2012 by the RCP to improve detection of, and response to, clinical deterioration in patients who are acutely ill. ${ }^{2}$

NEWS2, released in 2017, ${ }^{2}$ has been endorsed by NHS England and NHS Improvement for use in both the ambulance service and in acute hospitals. ${ }^{3,4}$ Three-quarters of acute trusts and all ambulance trusts are currently using it. ${ }^{3}$ NEWS has standardised how acute illness is assessed and responded to and, in hospitals alone, it is estimated that close to 2,000 deaths a year will be prevented. ${ }^{2}$

\section{An overview of NEWS2}

With NEWS2, a simple scoring system is used whereby a score is allotted to each of six physiological measurements (Box 1), which are commonly undertaken in healthcare settings. ${ }^{3}$ It has been validated for use in adults ( $>16$ years of age) but not in pregnant women or children $\left(<16\right.$ years of age)., ${ }^{2,3}$

The scores are calculated using the NEWS2 observation chart (Fig. 1). A score is allocated to each of the physiological measurements (Box 1); the higher the score, the more abnormal the measurement. ${ }^{3}$ The score is aggregated and,
Box 1 Six simple physiological measurements included in the scoring system in NEWS2 ${ }^{2}$

Respiratory rate

Oxygen saturations

Temperature

Systolic blood pressure

Pulse rate

Level of consciousness. 


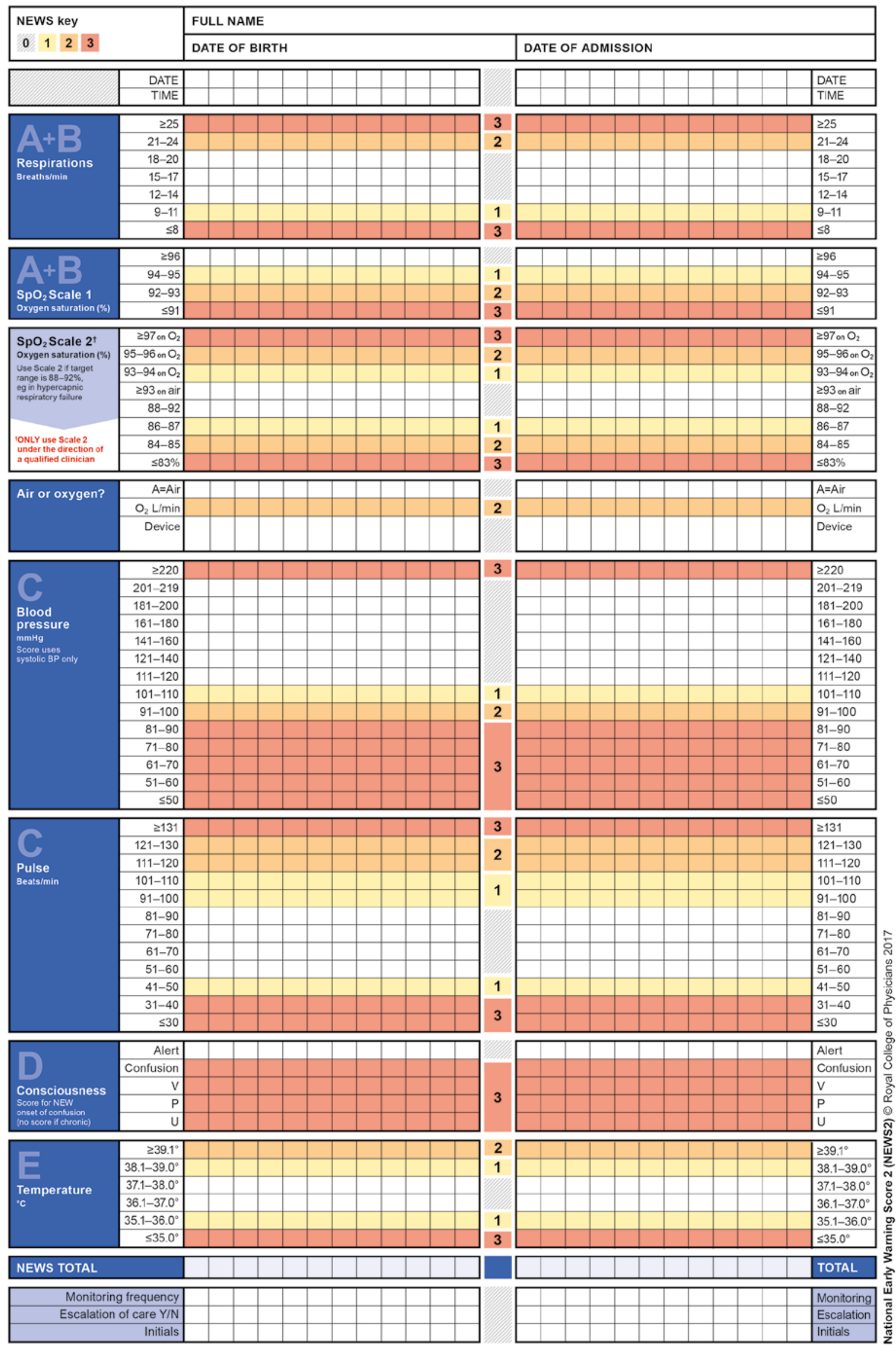

Fig. 1 NEWS2 observation chart (reproduced from Royal College of Physicians, National Early Warning Score (NEWS) 2: standardising the assessment of acute-illness severity in the NHS, updated report of a working party, London: RCP, 2017) ${ }^{2}$ 
if the patient requires supplementary oxygen, it is increased. An elevated NEWS score doesn't provide a diagnosis, but helps to identify patients who are sick, requiring urgent clinical review following a standardised approach. ${ }^{3}$

The NEWS thresholds and triggers chart (Fig. 2) can then prompt an appropriate response. Another chart providing guidance on the recommended clinical responses to NEWS trigger thresholds is available, particularly for use in hospital settings.

The NEWS2 observation chart (Fig 1) incorporates a number of improvements made to the first NEWS chart published in 2012, including:

- The familiar and systematic 'airway breathing circulation disability exposure' (ABCDE) approach advocated by the Resuscitation Council UK to assess (and treat) the acutely ill patient

- A list of ranges for the boundaries of each measurement score

- A specific section (SpO2 Scale 2) to be used when the patient has hypercapnic respiratory failure (usually COPD) who has a clinically recommended oxygen saturation measurement of $88-92 \%$

- A new 'confusion' (disorientation, delirium or any new alteration to mentation) component has been incorporated into the familiar AVPU assessment tool, which is now ACVPU (where 'C' represents confusion). This can help to identify suspected sepsis

- Improved section for the administration of oxygen

- The importance of considering sepsis is emphasised in patients who are known to have an infection, suspected to have an infection or are at risk of infection; in these patients, a NEWS2 score of 5 or more is the key trigger threshold.

\section{Benefits of using NEWS2 in primary care}

The RCP hopes that NEWS2 will be validated for use in primary care, assisting triage and communication of acute-illness severity to ambulance and hospital services. ${ }^{2}$ NHS England encourages the use of NEWS2 to assist clinical assessment in the primary care setting, as work continues to collect reliable evidence to validate its use. ${ }^{3}$

It has shown that the use of NEWS in the pre-hospital setting may facilitate earlier recognition of deteriorating patients, earlier involvement of senior emergency

\section{Chart 2: NEWS thresholds and triggers}

\begin{tabular}{|c|c|c|}
\hline NEW score & Clinical risk & Response \\
\hline Aggregate score 0-4 & Low & Ward-based response \\
\hline Red score & Low-medium & Urgent ward-based response* \\
\hline Score of 3 in any individual parameter & Medium & Key threshold for urgent response* \\
\hline Aggregate score 5-6 & High & Urgent or emergency response** \\
\hline
\end{tabular}

- Response by a clinician or team with competence in the assessment and treatment of acutely ill patients and in recognising when the escalation of care to a critical care team is appropriate.

"The response team must also include staff with critical care skills, including airway management.

Fig. 2 NEWS thresholds and triggers chart (reproduced from: Royal College of Physicians, National Early Warning Score (NEWS) 2: standardising the assessment of acute-illness severity in the NHS, updated report of a working party, London: RCP, 2017) ${ }^{2}$

department staff and more appropriate levels of critical care. ${ }^{5}$ Unsurprisingly, very high and very low NEWS scores are reliable indicators to identify patients who are and are not likely to deteriorate in the pre-hospital setting. ${ }^{6}$

Higher NEWS scores have been shown to be associated with decreased time from referral to arrival for patients conveyed by ambulance, together with decreased time from arrival in hospital to doctor review. ${ }^{7}$

A number of doctors' surgeries (West of England, Liverpool and Wessex) have found the use of NEWS helpful as an adjunct to decision-making, a prompt to do a complete set of observations and an aid to communication with the rest of the healthcare pathway, including the ambulance service, regarding deteriorating patients. ${ }^{3}$ This enables colleagues in other settings (for example, ambulance service and emergency department) to plan, prioritise and place patients safely and appropriately.

In summary, what NEWS2 offers is a prompt to encourage the reliable taking of physiological observations, an adjunct to clinical decisionmaking and a very clear, easily communicated and well-understood physiological score that the rest of the care pathway understands. ${ }^{8}$

\section{Benefits of using NEWS2 in the dental practice}

Although NEWS2 has not yet been validated for use in the dental practice setting, there are a number of reasons why dental practices should seriously consider introducing it as an adjunct to help them respond to a medical emergency: - NEWS2 can provide an objective assessment of a patient's physiological state, adding to and reinforcing clinical judgement; it can help reinforce dental teams' clinical findings and flag up patients who are more likely to deteriorate when having a medical emergency in the dental practice (particularly when consecutive NEWS2 scores display an upward trend)

- The NEWS2 observation chart (Fig. 1) reinforces the $\mathrm{ABCDE}$ approach to assess (and treat) the acutely ill patient

- NEWS2 can improve the objective assessment of patients with a medical emergency such as suspected sepsis, acute asthma attack and anaphylaxis

- NEWS2 can help with communication when calling 999 for an ambulance (every ambulance service in the UK now use it) ${ }^{3}$ because the NEWS2 score enables the dental team to communicate vital signs data in a common language

- NEWS2 can empower the dental team to be listened to; for example, when calling 999 for an ambulance, if they have concerns about a patient ${ }^{3}$

- The earlier a complete set of observations is done (for example, in the dental practice), the sooner a patient can be placed on a track and trigger score, enabling deterioration to be tracked and resources prioritised ${ }^{9}$

- In the current NHS climate where 'ambulance stacking' seems to be becoming more common, every bit of non-subjective information such as a high NEWS score can improve the triage, speed up the arrival of the paramedics and guide the urgency of transfer to the emergency department ${ }^{8}$

- NEWS2 can be used for communication and handover when patients are transferred to and from acute hospitals 


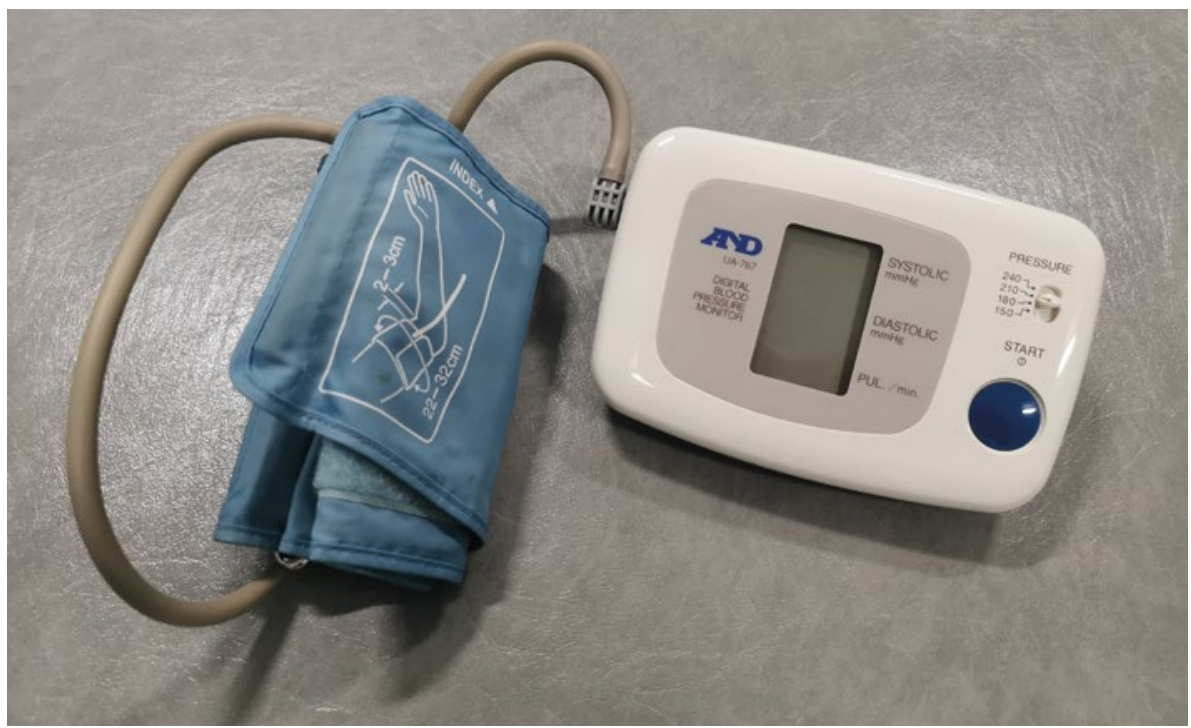

Fig. 3 Blood pressure monitor

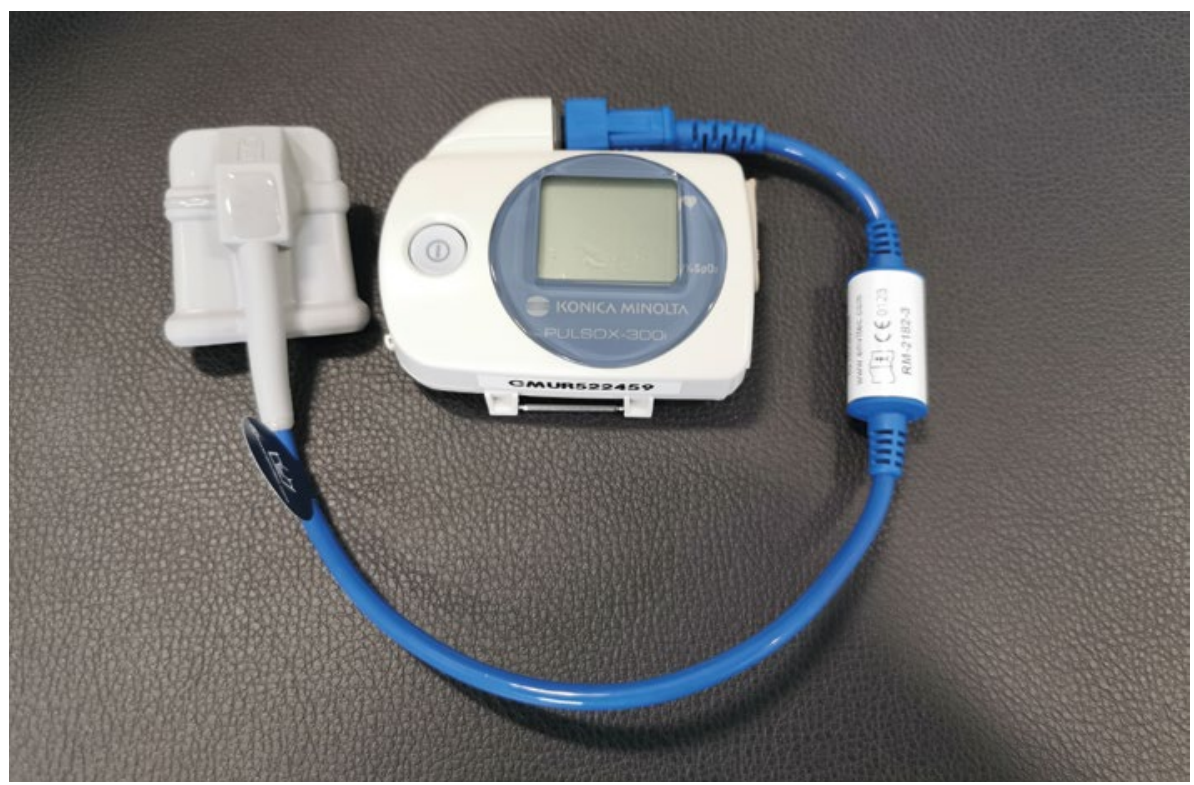

Fig. 5 Finger pulse oximeter

- A completed NEWS2 observation form is an excellent documentation record, providing the dental team with justification for what they did or didn't do if their actions are called into question

- NEWS2 can help with the objective assessment of an ill patient with suspected COVID-19 infection in the dental practice. $^{10}$

\section{Cautions}

It is important to remember that NEWS2 does not replace clinical judgement. ${ }^{9}$ Clinical judgement should always be used, even if the NEWS2 score is normal.

patients for review whenever they are concerned, even if the NEWS2 appears to be reassuring. A good example of this is a patient with chest pain. A patient could be having an acute coronary syndrome (heart attack) but have a normal NEWS2 score.

\section{Implementing NEWS2 into the dental practice}

Guidance and advice on implementing NEWS2 are freely available on the RCP's website (www. rcp.org.uk). In addition, some basic equipment together with appropriate staff training will be required.
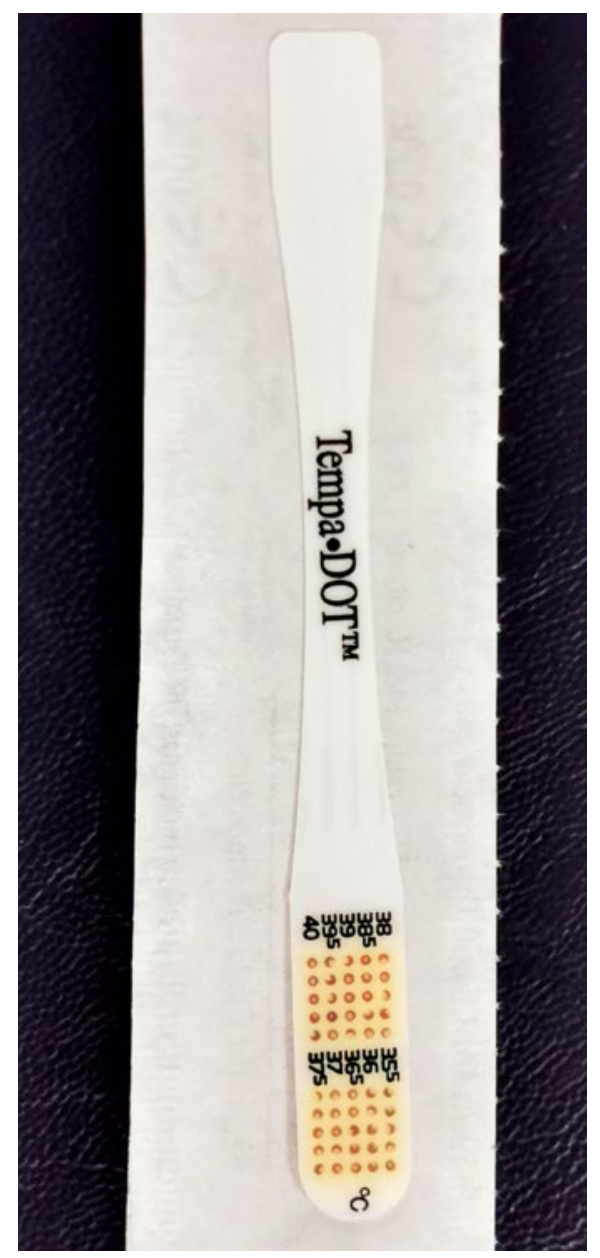

Fig. 4 Thermometer

\section{Equipment/resources}

The following equipment/resources will be required:

- Blood pressure monitor: at present, only dental practices that use sedation are required to have a blood pressure monitor (Fig. 3) for oxygen saturation monitoring. ${ }^{11}$ Some other dental practices already have one on the premises, but for those that don't, basic affordable devices are widely available
- Thermometer: dental practices should already have a thermometer (Fig. 4) because, according to NICE guidance, the confirmation of fever in a patient with a dental abscess is one indication for prescribing antibiotics. ${ }^{12}$ Also, due to the current crisis, a thermometer would be useful even more so now, and highly indicated in recording the temperature in a patient with suspected COVID-19 infection and managing our patients effectively

- Pulse oximeter: at present, only dental practices that use sedation are required to have a pulse oximeter (Fig. 5) for 
oxygen saturation monitoring. ${ }^{11}$ However, affordable fingertip pulse oximeters can be purchased and these devices are particularly useful in monitoring patients with medical emergencies such as sepsis, acute asthma attack and respiratory difficulties that can complicate COVID-19

- NEWS2 charts: freely available from the RCP's website. They are free to use, but it is not permitted to alter them and the charts need to be printed out in colour. ${ }^{2}$

\section{Training}

As well as having training in the use of the above devices, staff training and familiarisation with NEWS2 will be required. The RCP provides information relating to NEWS2 training resources on its website. The e-learning course ('Standardising the assessment of acute-illness severity in the NHS') is free for NHS staff with an NHS e-mail address (nhs.net or nhs.uk).

A training document together with webbased educational tools in varying formats to support NEWS2 being implemented at local level are available at http://tfinews. ocbmedia.com. ${ }^{2}$ These resources include using supplementary oxygen, the importance of new confusion and how NEWS2 can be used to recognise sepsis. ${ }^{2}$
In addition, NEWS2 refresher/awareness training can be simply included in annual medical emergencies training undertaken in dental practices.

\section{Conclusion}

Using NEWS2 in the dental practice will help members of the dental team effectively, confidently and safely manage medical emergencies, should they arise. This will facilitate effective teamwork and ensure enhanced patient outcomes. Ultimately, in line with GDC principle 1, the dental team will be working in the best interests of patients. ${ }^{13}$ This article has provided an overview to NEWS2 as well as some guidance on how to implement it.

\section{References}

1. General Dental Council. Scope of practice. 2013 Available at https://www.gdc-uk.org/docs/defaultsource/scope-of-practice/scope-of-practice.pdf (accessed August 2020).

2. Royal College of Physicians. National Early Warning Score (NEWS) 2: standardising the assessment of acute-illness severity in the NHS. 2017. Available online at https://www.rcplondon.ac.uk/projects/outputs/ national-early-warning-score-news-2 (accessed August 2020).

3. NHS England. National Early Warning Score (NEWS). 2017. Available at https://www. england.nhs.uk/ourwork/clinical-policy/sepsis/ nationalearlywarningscore/ (accessed May 2020).
4. Royal College of Physicians. NHS England approves use of National Early Warning Score (NEWS) 2 to improve detection of acutely ill patients. 2017. Available at https://www.rcplondon.ac.uk/news/nhs-englandapproves-use-national-early-warning-score-news-2improve-detection-acutely-ill (accessed May 2020).

5. Silcock D, Corfield A, Gowens P, Rooney K. Validation of the National Early Warning Score in the prehospital setting. Resuscitation 2015; 89: 31-35.

6. Patel R, Nugawela M, Edwards $\mathrm{H}$ et al. Can early warning scores identify deteriorating patients in prehospital settings? A systematic review. Resuscitation 2018; 132: 101-111.

7. Scott L, Redmond N, Tavaré A et al. Association between National Early Warning Scores in primary care and clinical outcomes: an observational study in UK primary and secondary care. Br J Gen Pract 2020; DOI: 10.3399/ bjgp20X709337.

8. Inada-Kim M, Nsutebu E. NEWS 2: an opportunity to standardise the management of deterioration and sepsis. BMJ 2018; 360: k1260.

9. Marsden R. The updated National Early Warning Score and its use with suspected sepsis. 2018. Available at https://www.rcgp.org.uk/clinical-and-research/about/ clinical-news/2018/january/the-updated-nationalearly-warning-score-and-its-use-with-suspectedsepsis.aspx (accessed May 2020).

10. Royal College Physicians. NEWS2 and deterioration in COVID-19. 2020. Available at https://www.rcplondon. ac.uk/news/news2-and-deterioration-covid-19 (accessed May 2020).

11. CQC. Dental mythbuster 10: Safe and effective conscious sedation. 2018. Available at https://www. cqc.org.uk/guidance-providers/dentists/dentalmythbuster-10-safe-effective-conscious-sedation (accessed May 2020).

12. NICE. Dental abscess. 2018. Available online at https://cks. nice.org.uk/topics/dental-abscess/ (accessed May 2020).

13. General Dental Council. Standards for the dental team. 2013. Available at https://standards.gdc-uk.org/Assets/ pdf/Standards\%20for\%20the\%20Dental\%20Team.pdf (accessed May 2020). 